\title{
Letter to the editor to update the article "Remote monitoring for heart failure using implantable devices: a systematic review, meta-analysis, and meta-regression of randomized controlled trials"
}

\author{
Alexander G. Hajduczok ${ }^{1}$ (D) . Samer N. Muallem ${ }^{2} \cdot$ Matthew S. Nudy $^{2} \cdot$ Ami L. DeWaters $^{3} \cdot$ John P. Boehmer $^{2}$ (D)
}

Accepted: 2 November 2021 / Published online: 9 November 2021

(c) The Author(s), under exclusive licence to Springer Science+Business Media, LLC, part of Springer Nature 2021

\begin{abstract}
Our recently published systematic review and meta-analysis of heart failure (HF) remote monitoring using implantable devices (Hajduczok et al. in HF Reviews 1-20, 1) has been updated to reflected new data from the GUIDE-HF trial (Lindenfeld et al. in Lancet 398(10304):991-1001, 2). Data from randomized controlled trials (RCTs) was assessed to determine the effectiveness of implantable remote monitoring on the improvement of outcomes in HF patients. With the inclusion of the data from 1000 patients followed for 12 months in GUIDE-HF, our conclusions remain unchanged: Compared to standard of care, remote monitoring using implantable devices did not reduce mortality, CV, or HF hospitalizations. However, right ventricular/pulmonary pressure monitoring may reduce HF hospitalizations.
\end{abstract}

Keywords Heart failure $\cdot$ Remote monitoring $\cdot$ Implantable devices $\cdot$ Systematic review $\cdot$ Meta-analysis $\cdot$ Randomized controlled trials

To the editors:

We would like to update our recently published manuscript (Hajduczok et al. [1]) due to the emergence of new data from the GUIDE-HF randomized controlled trial (RCT) that meets the inclusion criteria of our systematic review and meta-analysis $[1,2]$.

Alexander G. Hajduczok

alexander.hajduczok@jefferson.edu

Samer N. Muallem

smuallem@pennstatehealth.psu.edu

Matthew S. Nudy

mnudy@pennstatehealth.psu.edu

Ami L. DeWaters

adewaters@pennstatehealth.psu.edu

1 Division of Cardiology, Jefferson Heart Institute, Sidney Kimmel School of Medicine, Thomas Jefferson University, 925 Chestnut St, Suite 200, Philadelphia, PA 19107, USA

2 Division of Cardiology, Penn State Health Milton S. Hershey Medical Center, Penn State College of Medicine, 500 University Drive, Hershey, PA H04717033, USA

3 Division of Internal Medicine, Penn State Health Milton S. Hershey Medical Center, Penn State College of Medicine, 500 University Drive, Hershey, PA 17033, USA
In Hajduczok et al., we reported data from 11 RCTs that tested implantable remote monitoring versus standard of care for management of heart failure (HF) patients [1]. Primary endpoints were all-cause mortality and a composite of cardiovascular (CV) and HF hospitalizations. Remote monitoring did not reduce mortality (RR 0.89 [95\% CI 0.77, 1.03]) or the composite of CV and HF hospitalizations (RR 0.98 $[0.81,1.19])$. Subgroup analysis found significant heterogeneity of treatment effects (HTE) for hospitalizations between those studies that used right ventricular/pulmonary pressure monitoring versus impedance-based monitoring $\left(I^{2}=87.1 \%\right.$, $\left.\mathrm{chi}^{2}=7.75, p=0.005\right)$. We concluded that although implantable remote monitoring did not reduce mortality, CV hospitalizations, or HF hospitalizations, that right ventricular/ pulmonary pressure monitoring may reduce HF hospitalizations and that these conclusions would need further validation from future studies, namely from GUIDE-HF [2].

GUIDE-HF, published in the Lancet in 2021, built upon the CHAMPION trial, which showed a $33 \%$ reduction in HF hospitalizations after 18 months of follow-up using pulmonary artery (PA) pressure monitoring versus control [3]. CHAMPION enrolled New York Heart Association (NYHA) Class III HF patients with a previous HF hospitalization but was not powered to detect differences in mortality. In GUIDE-HF, 1000 patients were randomized to a PA pressure monitoring 
strategy using CardioMEMS (Abbott) or standard follow-up for HF. The trial enrolled a broader group of patients than CHAMPION, including those with NYHA Class II-IV symptoms regardless of ejection fraction (EF) and either a HF hospitalization within 12 months or elevated BNP or NTproBNP $(B N P \geq 250 \mathrm{pg} / \mathrm{mL}$ or NT-proBNP $\geq 1000 \mathrm{pg} / \mathrm{mL})$. The primary endpoint was a composite of all-cause mortality and HF events (HF hospitalizations and urgent emergency department or unplanned clinic visits) [3].

The study did not meet its primary endpoint, with 253 primary endpoint events ( 0.56 events per patient-year) occurring in the remote monitoring group and 289 events (0.64 per patient-year) in the standard of care group (hazard ratio [HR] 0.88, 95\% confidence interval (CI) 0.74-1.05). There were $185 \mathrm{HF}$ hospitalizations in the treatment group and 225 in the control group (HR 0.83, 95\% CI 0.68-1.01). There were no significant differences in mortality between the treatment and control group [3].

In a pre-COVID-19 subgroup analysis comparing outcomes prior to March 2020, there was a reduction in primary endpoint events with 177 events $(0.55$ events per patient-year) in the treatment group and 224 events $(0.68$ events per patient-year) in the control group (HR 0.81, 95\% CI 0.66-1.00). Similarly, HF hospitalizations were reduced in the treatment group (HR $0.72,95 \%$ CI $0.57-0.92$; $p=0.0072$ ). Consistent with the overall analysis, there were no differences in all-cause mortality in this subgroup [3].

The addition of the GUIDE-HF results to our metaanalysis yields 12 total RCTs of implantable remote monitoring for HF patients $(n=7196)$ with mean follow-up of 20.5 months, mean age of 65 years, and EF of $29.3 \%$, respectively (Tables S1, S2). The overall results of our analysis are unchanged. There remains no difference in all-cause mortality (RR 0.91 [95\% CI 0.79-1.04]), CV or HF hospitalizations (RR 0.96 [95\% CI 0.82-1.14]), or HF hospitalizations alone RR 0.94 [95\% CI 0.77-1.15]) (Figs. S1-4).

Notably for HF hospitalizations, there remains a difference in treatment effects between those with implanted right ventricular or pulmonary pressure monitoring systems versus those with thoracic impedance-based monitoring devices (RR 0.77 [95\% CI 0.64-0.92] vs. RR 1.10 [95\% CI 0.96-1.26]; test for subgroup differences: $I^{2}=89.5 \%$, $\mathrm{chi}^{2}=9.56, p=0.002$ ) (Fig. 1).

The findings from GUIDE-HF are consistent with our prior conclusion that right ventricular/pulmonary pressure monitoring may reduce HF hospitalizations [1]. Overall the addition of the GUIDE-HF results to our previously published meta-analysis tightened the confidence interval and had minimally affected the point estimate for reduction of HF hospitalizations.

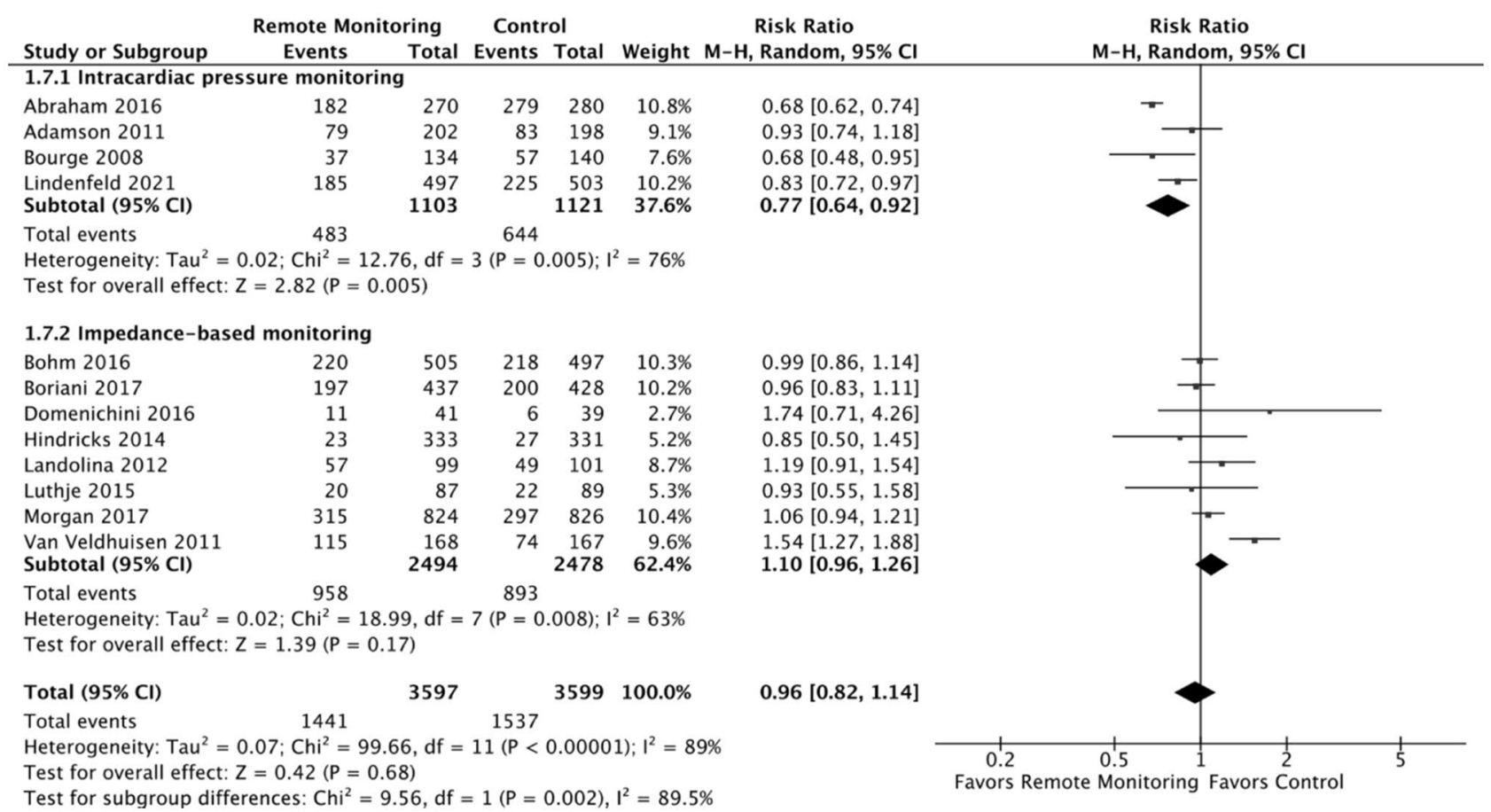

Fig. 1 Intracardiac pressure monitoring vs. thoracic impedance-based monitoring (subgroup analysis). Outcomes shown are the composite of $\mathrm{HF}$ or $\mathrm{CV}$ hospitalizations. Subgroup analysis showed there was a statistically significant difference in the composite of $\mathrm{CV}$ or $\mathrm{HF}$ hospitalizations when comparing studies using implanted right ven-

tricular/pulmonary pressure monitoring versus impedance-based monitoring, favoring the intracardiac pressure monitoring (RR 0.77 [0.64, 0.92] vs. RR $1.10[0.96,1.26])$; test for subgroup differences: $I^{2}=89.5 \%, \mathrm{chi}^{2}=9.56, p=0.002$ 
The reasons for the more modest treatment effect on HF hospitalizations in the GUIDE-HF trial in comparison to the CHAMPION trial will be debated. However, when the entire body of evidence for HF management with hemodynamic pressure monitoring is taken as a whole, the conclusion continues to suggest a possible benefit in reducing HF hospitalizations. However, when analyzing all studies, remote monitoring does not reduce the primary endpoint of all-cause mortality and HF hospitalizations. Additional work will be needed to discern the most appropriate use for improvement of outcomes using implantable remote monitoring strategies, but GUIDE-HF adds valuable information to this rapidly evolving field.

Supplementary Information The online version contains supplementary material available at https://doi.org/10.1007/s10741-021-10190-x.

\section{Declarations}

Conflict of interest John P. Boehmer, MD, is a consultant with Boston Scientific, Nanowear, Inc. and Medtronic and has research grants sponsored by each of those entities. All other authors have no conflicts of interest.

\section{References}

1. Hajduczok AG, Muallem SN, Nudy MS, DeWaters AL, Boehmer JP (2021) Remote monitoring for heart failure using implantable devices: a systematic review, meta-analysis, and meta-regression of randomized controlled trials. Heart Fail Rev 1-20. https://doi. org/10.1007/s10741-021-10150-5. Epub ahead of print. PMID: 34559368; PMCID: PMC8460850

2. Lindenfeld J, Zile MR, Desai AS, Bhatt K, Ducharme A, Horstmanshof D, Krim SR, Maisel A, Mehra MR, Paul S, Sears SF, Sauer AJ, Smart F, Zughaib M, Castaneda P, Kelly J, Johnson N, Sood P, Ginn G, Henderson J, Adamson PB, Costanzo MR (2021) Haemodynamic guided management of heart failure (GUIDE-HF): a randomised controlled trial. Lancet 398(10304):991-1001. https://doi.org/10.1016/ S0140-6736(21)01754-2. Epub 2021 Aug 27. PMID: 34461042

3. Abraham WT, Stevenson LW, Bourge RC, Lindenfeld JA, Bauman JG, Adamson PB, CHAMPION Trial Study Group (2016) Sustained efficacy of pulmonary artery pressure to guide adjustment of chronic heart failure therapy: complete follow-up results from the CHAMPION randomised trial. Lancet 387(10017):45361. https://doi.org/10.1016/S0140-6736(15)00723-0. Epub 2015 Nov 9. PMID: 26560249

Publisher's Note Springer Nature remains neutral with regard to jurisdictional claims in published maps and institutional affiliations. 